\title{
Measurements of event properties and correlations in multijet events in CMS
}

\author{
Hyunyong Kim*† \\ University of Seoul \\ E-mail: hyunyong.kimecern.ch
}

We present results on measurements of characteristics of events with jets, from jet-charge over investigations of shapes to jet mass distributions, and angular correlations in multi-jet events. The measurements are compared to theoretical predictions including those matched to parton shower and hadronization.

XXXIX International Conference on High Energy Physics

4-11 July 2018

COEX, Seoul, Republic of Korea

* Speaker.

${ }^{\dagger}$ on behalf of the CMS Collaboration. 


\section{Introduction}

The measurements of jet properties are important to the understanding of physics at hadron colliders and quantum chromodynamics (QCD). When the partons interact under the strong interaction, this interaction is described by QCD using perturbative techniques (pQCD). The measurement of angular correlation is an interesting tool to gain insight into multi-jet production processes. This angular correlation analysis shows the normalized inclusive 2-jet cross sections as a function of the azimuthal angular separation between the two leading $p_{\mathrm{T}}$ jets [1]. Another interesting property is the mass of the jet. The jet mass is more sensitive to the internal structure of jets theoretically described by QCD parton showering [2]. The measurement is performed using data collected with the CMS detector [3].

\section{Results and Conclusions.}

The event reconstruction is base on the CMS particle flow (PF) algorithm [4] which takes into account information from all sub-detectors. Charged hadrons associated with pileup vertices are removed before jet clustering. This method is referred to as charged hadron subtraction (CHS) [5]. Several theoretical predictions are compared to data.

The PYTHIA8 [6] is generated with CUETP8M1 tune [7]. The HERWIG++ [8] is generated with CUETHppS1 tune [7]. Both the PYTHIA8 and HERWIG++ are based on L0 $2 \rightarrow 2$ matrix element (ME) calculation. The MADGRAPH [9] LO $(2 \rightarrow 2+3+4)$ ME calculations include parton showering with PYTHIA8. The POWHEG $[10,11,12] 2$-jet and 3-jet are NLO ME calculations with PYTHIA8 or HERWIG++ parton showering. The HERWIG7 [13] uses NLO di-jet ME calculation within the MC@NLO [14] procedure through angular-ordering emissions.

The figure 1 (a) shows normalized inclusive 2-jet differential cross-sections in $\Delta \phi_{1,2}$ for various $p_{\mathrm{T}}^{\max }$ regions. The distributions are strongly peaked at $\pi$ and become perpendicular with increasing $p_{\mathrm{T}}^{\max }$. Comparison of the data to POWHEG 2jet + PYTHIA8 event generator. The error bars on the data points represent the total experimental uncertainty, which is the quadratic sum of the statistical and systematic uncertainties. The region away from $\pi$ is sensitive to hard radiation from ME calculation. Otherwise, the region close to $\pi$ is sensitive to resummation contributions from PS calculation. The figure 1 (b) shows the ratios of the PYTHIA8, HERWIG++, MADGRAHP+PYTHIA8 event generators predictions to the normalized inclusive 2-jet cross section differential in $\Delta \phi_{1,2}$ for all $p_{\mathrm{T}}^{\max }$. Among the LO di-jet event generators HERWIG++ exhibits the largest deviations from the measurements. The PYTHIA8 behaves much better than HERWIG++ exhibiting some deviations particular around $\Delta \phi=5 \pi / 6$. The MADGRAPH + PYTHIA8 event generator provides the best description of the measurements. The figure 1 (c) shows the ratios of the POWHEG 2 jet matched to PYTHIA8 and HERWIG++, POWHEG 3 jet + PYTHIA8, and HERWIG7 event generators predictions to the normalized inclusive 2-jet cross section differential in $\Delta \phi_{1,2}$ for all $p_{\mathrm{T}}^{\max }$. The predictions of POWHEG 2 jet or POWHEG 3 jet exhibit large deviations from the measurements. It has been checked that POWHEG 2 jet predictions at parton level give a reasonable description of the measurement for values of $\Delta \phi_{1,2}$ greater than $\approx 2 \pi / 3$, while they completely fail for smaller values, where the parton shower has a crucial role. Adding parton showers fills the phase space at low values of $\Delta \phi_{1,2}$ and brings the POWHEG 2 jet predictions closer to data. The predictions from 
POWHEG 2 jet matched to PYTHIA8 are describing the normalized cross sections better than those where POWHEG 2 jet is matched to HERWIG++. Since the hard process calculation is the same, the difference between the two predictions is entirely due to different parton shower in PYTHIA8 and HERWIG++, which also use different $\alpha_{S}$ values for initial and final-state emissions, in addition to a different upper scale used for the parton shower simulation, which is higher in PYTHIA 8 than in HERWIG++. The di-jet NLO event generator HERWIG7 provides the best description of these measurements, showing a very large improvement in comparison to HERWIG++. For this observable, the MC@NLO method of combining parton shower with the NLO parton level calculations has advantages compared to the POWHEG method.

The figure 2 shows comparing the normalized ungroomed and groomed cross sections, this results show that the grooming algorithm considerably lowers the jet mass and suppresses the Sudakov peak, as expected. The precision of the measurement improves, since the grooming algorithm removes the parts of the jet arising from soft radiation and the pileup contribution. At the figure 2 (c) and (d), the data are shown by the black points, with dark grey bands for the statistical uncertainty (Stat. unc.) and with light grey bands for the total uncertainty (Stat.+sys. unc., added in quadrature). The LO theory predictions with a MC-based physics model are from PYTHIA 8 and HERWIG++, and they predict the ungroomed jet mass measurement within uncertainties. Above the splitting threshold $(m / p t>0.3)$, the prediction is slightly larger than the data. After grooming some deviations from the data can be observed at very low jet masses. The NLO theory prediction with a MC-based physics model is from POWHEG + PYTHIA8, and is observed to have largely the same behavior as PYTHIA8 alone, so the largest effects are coming from the physics model. The LO and NLO theory predictions with analytic resummation agree overall with the data, with some slight disagreements at very low masses.

\section{References}

[1] CMS collaboration, A. M. Sirunyan et al., Azimuthal correlations for inclusive 2-jet, 3-jet, and 4-jet events in pp collisions at $\sqrt{s}=13$ TeV, Eur. Phys. J. C78 (2018) 566 [1712.05471].

[2] CMS collaboration, C. Collaboration, Measurement of the differential jet production cross section with respect to jet mass and transverse momentum in dijet events from pp collisions at $\sqrt{\mathrm{s}}=13 \mathrm{TeV}$,

[3] CMS collaboration, S. Chatrchyan et al., The CMS Experiment at the CERN LHC, JINST 3 (2008) S08004.

[4] CMS collaboration, A. M. Sirunyan et al., Particle-flow reconstruction and global event description with the CMS detector, JINST 12 (2017) P10003 [1706. 04965].

[5] CMS collaboration, C. Collaboration, Pileup Removal Algorithms, .

[6] T. Sjöstrand, S. Ask, J. R. Christiansen, R. Corke, N. Desai, P. Ilten et al., An Introduction to PYTHIA 8.2, Comput. Phys. Commun. 191 (2015) 159 [1410.3012].

[7] CMS collaboration, V. Khachatryan et al., Event generator tunes obtained from underlying event and multiparton scattering measurements, Eur. Phys. J. C76 (2016) 155 [1512.00815].

[8] M. Bahr et al., Herwig++ Physics and Manual, Eur. Phys. J. C58 (2008) 639 [0803. 0883].

[9] J. Alwall, M. Herquet, F. Maltoni, O. Mattelaer and T. Stelzer, MadGraph 5 : Going Beyond, JHEP 06 (2011) 128 [1106. 0522]. 
[10] P. Nason, A New method for combining NLO QCD with shower Monte Carlo algorithms, JHEP 11 (2004) 040 [hep-ph/ 0409146$].$

[11] S. Frixione, P. Nason and C. Oleari, Matching NLO QCD computations with Parton Shower simulations: the POWHEG method, JHEP 11 (2007) 070 [0709.2092].

[12] S. Alioli, P. Nason, C. Oleari and E. Re, A general framework for implementing NLO calculations in shower Monte Carlo programs: the POWHEG BOX, JHEP 06 (2010) 043 [1 002 . 2 581].

[13] J. Bellm et al., Herwig 7.0/Herwig++ 3.0 release note, Eur. Phys. J. C76 (2016) 196 [1512 . 01178$].$

[14] S. Frixione and B. R. Webber, Matching NLO QCD computations and parton shower simulations, JHEP 06 (2002) 029 [hep-ph/ 0204244$].$

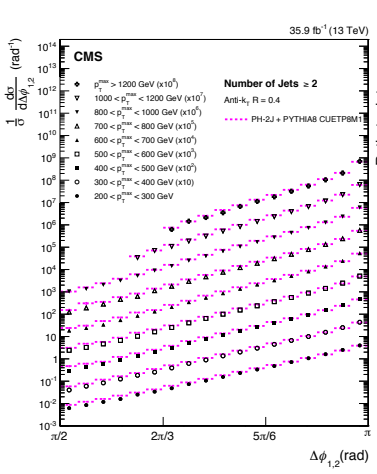

(a)

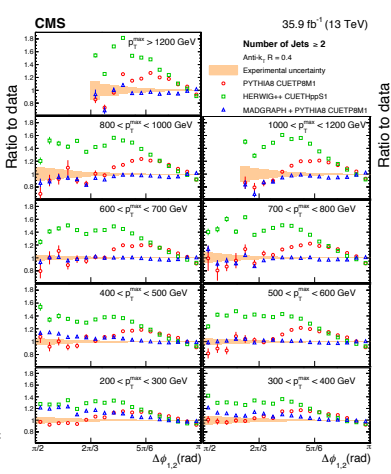

(b)

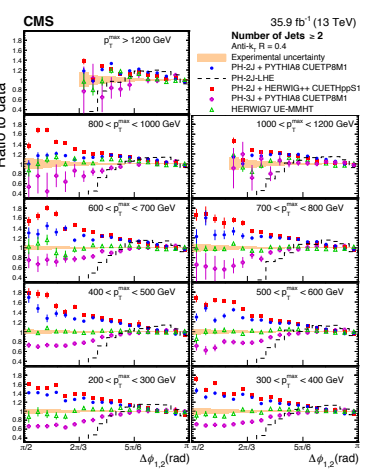

(c)

Figure 1: (a) Normalized inclusive 2-jet cross section differential in $\Delta \phi_{1,2}$ for nine $p_{\mathrm{T}}^{\max }$ regions, scaled by multiplicative factors for presentation purposes. The size of the data symbol includes both statistical and systematic uncertainties. (b, c) Ratios of theoretical predictions to the data. The solid band indicates the total experimental uncertainty and the vertical bars on the points represent the statistical uncertainties in the simulated data.

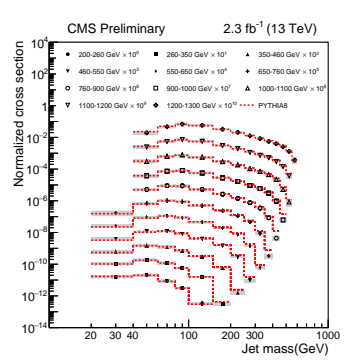

(a)

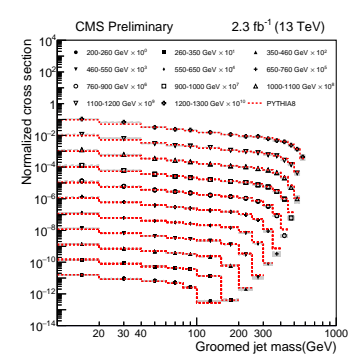

(b)

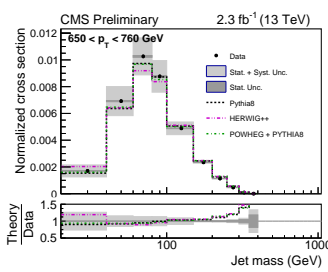

(c)

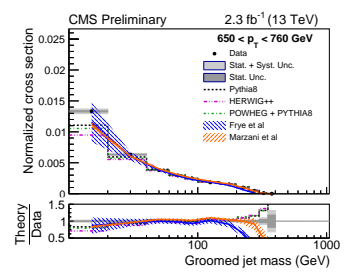

(d)

Figure 2: (a) Results of unfolding ungroomed jets for all $p_{\mathrm{T}}$ bins. (b) Results of unfolding groomed jets for all $p_{\mathrm{T}}$ bins. (c) Results of unfolding the ungroomed jets for $650<p_{\mathrm{T}}<760$ bins. (d) Results of unfolding the groomed jets for $650<p_{\mathrm{T}}<760$ bins. 